\title{
Profesorado y familia en la realidad educativa intercultural. Discursos y prácticas
}

\author{
Teacher and family into the intercultural realities. Speeches and practices
}

\author{
Eva María González-Barea / evamgon@um.es \\ Universidad de Murcia, España
}

\begin{abstract}
This article analyzes the participation of families in the school, inquiring about the specific problems that they can present the foreign families, acting as border elements to effective communication between school, teachers and family. The objectives that responds the article are: 1) analyze the way in which foreign and native families participate in schools, as well as, 2) the activities organized since the centres with the intention of promoting participation. An ethnographic methodological approach, the collection of data has been done in two geographical contexts, Seville and Jaén, in which a case study has been developed in a school of each of the above cities. In the light of the data obtained, stresses that the participation of families in schools not depends directly on the foreignness of the same, but the family interest in education and school and practices and actions implemented since the schools to provide equal access to participation.
\end{abstract}

Key words: school, teacher, family, participation, inmigrants.

Resumen: En este artículo se analiza la participación de las familias en la escuela, indagando sobre las problemáticas específicas que pueden presentar las familias extranjeras, actuando como elementos fronterizos para una eficaz comunicación entre escuela, profesorado y núcleo familiar. Los objetivos a los que responde el artículo son: 1) analizar el modo como las familias autóctonas y extranjeras participan en el ámbito escolar, así como 2) conocer las actividades organizadas desde los centros con la intención de promover la participación. Con un enfoque metodológico etnográfico, la recogida de los datos se ha realizado en dos contextos geográficos: Sevilla y Jaén, donde se ha desarrollado un estudio de caso en un centro educativo de cada una de las ciudades citadas. A la luz de los datos obtenidos, se hace hincapié en que la participación de las familias en los centros educativos no depende directamente de la extranjeridad de las mismas, pero sí del interés familiar por la educación y la escuela y las prácticas y actuaciones puestas en marcha desde los colegios para posibilitar el acceso igualitario en la participación.

Palabras clave: escuela, profesorado, familia, participación, población inmigrante. 


\section{Familia, escuela e inmigración. Participación familiar en la escuela}

La participación familiar en la escuela, con especial énfasis en las familias migrantes, y la gestión de la diversidad cultural en los contextos escolares bajo la filosofía defendida por el paradigma intercultural dan forma al marco teórico donde se ubica este trabajo. Concretamente, el interés del presente artículo radica en el análisis y reflexión sobre el modo en el que las familias extranjeras participan en el ámbito escolar, indicando las diferentes casuísticas que impiden su presencia más "normalizada" en el centro educativo, conociendo, a su vez, las actividades organizadas desde los colegios que intentan promover la participación familiar en clave "intercultural".

Atendiendo al concepto de participación y su significación, éste implicaría una "intervención, real y directa: tomar parte, ser protagonista de la propia vida y no sólo espectador o destinatario de las iniciativas ajenas, influir en la toma de decisiones que le afectan a uno mismo y al colectivo del que formamos parte" (De la Guardia y Luengo, 2007: 263). A su vez, la participación familiar en los centros educativos debe aparecer como un elemento más a tener en cuenta en la consecución de los objetivos educativos, siendo un factor importante en el marco de la función educativa de la propia escuela.

En esta línea, Pozuelos (2008: 130) indica que la participación implica tomar parte activa en el proceso de la educación y para ello se suele recurrir a dos estrategias: "1) implicar a los padres directamente en algunas experiencias de aula: talleres, charlas, salidas, etc. y 2) hacer explícito el plan de trabajo a los padres y madres de manera que puedan actuar desde el ámbito doméstico en el desarrollo de ciertos objetivos y actividades de carácter socio-familiar estrechamente relacionados con los didácticos del aula".

Por tanto, en el interés por "favorecer la convivencia entre familia y escuela se hace necesario que el centro educativo abra sus puertas a la comunidad, especialmente a los padres" (Hernández y López, 2006: 20). Sin embargo, "la participación de las familias en la escuela no acaba de despegar como un elemento sustancial entre los distintos miembros de las comunidades educativas" (Essomba, 2008: 138). En el caso que nos ocupa más concretamente, la participación de las familias inmigrantes, García Castaño, Rubio Gómez y Bouachra (2008: 39) indican que algunos investigadores consideran que éstas "son construidas desde la escuela, por el desinterés por la escolarización de sus hijos e hijas y la responsabilidad de los resultados que sus hijos e hijas obtienen en la escuela”. Sin embargo, es imprescindible no caer en explicaciones y reflexiones homogéneas al referirse a la participación de familias inmigrantes en los contextos escolares, ya que la población inmigrante de hoy 
presenta heterogeneidades que hacen difícil las generalizaciones respecto a ello (Rodríguez, 2011).

A pesar de eso, cuando se aborda esta cuestión sale a colación una rápida problematización de la participación familiar en el ámbito educativo vinculada a su origen no nacional, al desconocimiento e incorrecto manejo de la lengua, la impericia de los aspectos administrativos, la falta de tiempo e incompatibilidad horaria con sus trabajos y un especial acento en su supuesta no participación escolar (González Barea, 2008).

Entre los tópicos utilizados para explicar el fracaso e inadaptación de los menores en la escuela son cada vez más frecuentes los que aluden al hipotético "conflicto entre tradicionalismo de los protagonistas y la modernidad de los/as hijos/as, la supuesta identidad doble o dividida de éstos, o la creencia ingenua de que todos sus problemas escolares se solucionarían con la implementación del currículo intercultural" (García Borrego, 2008: 131).

Esto es, nuevamente insistiendo en que el problema está en ellos, los menores y sus familias, y la cultura que les define y condiciona, enfatizando sus especificidades etno-culturales. Por un lado, se obvian en las explicaciones aspectos como "las condiciones que comparten con otros sectores de las clases populares autóctonas en razón de su estatus social subordinado" (García Borrego, 2008: 132); y por otro, se tiende a evitar analizar las estrategias educativas de las familias y sus expectativas sobre la escuela.

Comprender, por tanto, el papel que asumen o se les otorga a las familias requeriría, por una parte, atender al modo en el que estas familias participan en el ámbito escolar, el modo como piensan la interculturalidad, las maneras en las cuales consideran a sus hijas e hijos portadores de diversidad (o no) y les educan y socializan en la configuración de las diferencias. Y por otra, deviene imprescindible explorar también la importancia que el profesorado concede a las familias, especialmente las extranjeras, para explicar la integración, el éxito y fracaso escolar (Colectivo IOE, 2007) o los alcances de determinadas prácticas de interculturalidad y su vinculación entre los colegios, el profesorado y las familias.

En este sentido, partimos de una concepción de lo intercultural desde una mirada múltiple, considerando que en la escuela hacer referencia a la interculturalidad pasa por entenderla como:

un enfoque educativo basado en el respeto y valoración de la diversidad cultural, dirigido a todos y cada uno de los miembros de la sociedad en su conjunto que propone un modelo de intervención, formal e informal, holístico, integrado, configurador de todas las dimensiones del proceso educativo en orden a lograr la igualdad de oportunidades/ resultados, la superación del racismo en sus diversas manifestaciones, la comunicación y las competencias interculturales (Aguado, 1996: 54). 
Por tanto, la interculturalidad necesita ser conceptualizada como "una reforma escolar en sentido amplio” (Nieto, 1992: 207), que reconozca la importancia de la participación de las familias y el contexto dentro del ámbito escolar, tal y como defienden los postulados del enfoque intercultural crítico sistematizado por Gorski (2009) y que defendemos desde este trabajo.

El trabajo que se presenta da cuenta de dichos postulados en la práctica educativa, indagando en las problemáticas y limitaciones que muestran las familias inmigrantes para ejercer una participación real en la escuela donde sus hijos/as se encuentran escolarizados/as.

Para ello, se parte de los significados y comportamientos que surgen en la articulación entre escuela, interculturalidad y participación familiar, donde el factor "extranjería" podría ser influyente en el nivel de participación de las familias, pues "las familias inmigrantes además de estar sometidas a los factores generadores de inestabilidad y a las presiones hacia la desintegración que afectan a todas las familias" (Beck, 1998); lo están, además, a procesos que les son específicos y tienen que ver con fragmentaciones y recomposiciones que afectan a las relaciones familiares y determinan las condiciones de socialización de sus hijos (García, 2008).

En estas realidades, los centros educativos suponen un territorio necesario de analizar, incluyendo además de la perspectiva de las propias familias inmigrantes, las del profesorado y la de las familias autóctonas sobre el eje transversal de "lo intercultural". Es precisamente esa mirada hacia la familia en los contextos escolares la que se plantea en este artículo, atendiendo especialmente a la realidad de las familias extranjeras y sus problemáticas añadidas para verse reflejadas en las dinámicas escolares.

\section{Metodología y proceso de investigación}

El proceso de investigación que se ha desarrollado en este trabajo se enmarca dentro de lo meramente etnográfico, entendiendo la etnografía como el estudio que trata de captar significados y reglas de acción social en un contexto particular contando con una interacción constante con los y las informantes (Velasco y Díaz de Rada, 1997).

$\mathrm{Al}$ mismo tiempo, no se ha tratado de una investigación que propone conocer para evaluar o comprobar que se dice lo que se hace en materia de educación intercultural. Nuestro posicionamiento etnográfico estriba más bien, como explica Bertely (2001: 34), en relación con los retos de la etnografía en educación, "en comprender, desde dentro y en situaciones específicas, las representaciones sociales -oficiales y no oficiales, escritas u orales, informadas 
o fundadas en la opinión pública- que conforman el entramado cultural de la educación escolarizada".

$\mathrm{Y}$ añade, "esto no implica encontrar una verdad, sino inscribir e interpretar las múltiples verdades que ponen en duda la legitimidad del discurso escolar hegemónico y que permiten desentrañar las lógicas discursivas, las producciones y amalgamas significativas, las fisuras y vacíos ocultos tras una racionalidad aparente, así como las voces silenciadas que conforman la cultura escolar".

Así, este trabajo se caracteriza por su carácter exploratorio, descriptivo e inductivo, ya que no se parte de la elaboración y comprobación de hipótesis sino de la interpretación -en su sentido más antropológico (Wolcott, 1993) - y el análisis a partir de los discursos de los actores informantes: profesorado y familias, en este caso.

La investigación en la que se enmarca el presente trabajo ha tenido una duración de tres años (2006-2009), teniendo en cuenta para su realización distintos contextos y actores. Respecto a los contextos de estudio, éstos corresponden a dos capitales de provincia de la comunidad andaluza en el sur de España: Sevilla y Jaén. La elección de estos dos contextos radica en la configuración de dos escenarios que se presentan muy diversos en lo que a instalación de extranjeros/as se refiere, y por ende, en lo que a conformación de las percepciones sobre diversidad conlleva: Jaén ofrece una zona de carácter urbano con una fuerte influencia del contexto rural (la principal presencia de población extranjera inmigrante que llega a la ciudad viene producida por la mano de obra que demanda el sector agrícola), frente a un contexto de carácter más urbano como es el de la ciudad de Sevilla.

Respecto a la presencia de población escolar extranjera cabe indicar que Jaén es la provincia andaluza con más bajo porcentaje de población escolar extranjera -un 3,5\% de alumnado extranjero-, frente a Sevilla, una de las provincias que presenta mayor porcentaje de alumnado extranjero - un 12,1\%- (según el Instituto de Estadística de Andalucía para el curso académico 2009-2010).

En ambas ciudades se ha realizado un estudio de caso concreto en un centro educativo. Los dos colegios han sido seleccionados de forma intencional. Ambos comparten una característica de interés para la investigación: la "relativamente alta" presencia de población escolar extranjera en el centro escolar respecto a la distribución total en cada una de las ciudades. 


\section{Descripción de los centros escolares seleccionados}

Colegio de Sevilla. Este centro educativo de carácter público se encuentra en el distrito de Cerro-Amate en la ciudad de Sevilla, que se define como una de las principales zonas de expansión de la ciudad, con nuevas áreas tanto residenciales como industriales.

El perfil sociofamiliar en la zona es muy variado: esta barriada se caracteriza por constar de familias monoparentales con hijos a cargo, situación económica muy baja, bajo nivel socioeducativo, trabajos esporádicos o dedicación a la economía sumergida, problema endémico de adicciones de diverso tipo por parte de uno o varios de los miembros familiares y con una escasa red de apoyo o con las relaciones extensas muy deterioradas.

En términos educativos, para el curso 2008/2009, el centro cuenta con 242 alumnos/as, 190 aparecen teniendo como país de origen España, 6 de Bolivia, 6 de China, 1 de Colombia, 1 de Ecuador, 10 de Marruecos, 1 de Perú, 24 de Rumania, 2 de Rusia y 1 de Venezuela, lo cual implica que hay un total de 52 alumnos cuyo país de origen no es España (21,48\%). En el curso citado, 21 alumnos están diagnosticados como de Necesidades Educativas Especiales, dos de ellos teniendo como país de origen Rumania, aunque uno de éstos ha sido desplazado a otro centro escolar.

En el curso 2009/2010 el centro cuenta con 241 alumnos/as matriculados, de los cuales 44 son alumnos/as procedentes de familias extranjeras; esto representa el 18,25\% del total del alumnado matriculado. Distinguiendo por nacionalidades aparecen: 3 de Bolivia, 6 de China, 3 de Colombia, 2 de Ecuador, 10 de Marruecos, 1 de Perú, 17 de Rumania, 1 de Rusia, 1 de Venezuela.

Se puede apreciar el descenso de alumnado extranjero en el actual curso académico como consecuencia de varios factores: en primer lugar, porque varias familias se marcharon del barrio a finales del curso pasado como consecuencia de la crisis económica y la carencia de empleo; en segundo lugar, los dos grupos de sexto curso de Educación Primaria del curso pasado concentraban un alto índice de alumnado procedente de familias extranjeras. Por lo tanto, el salto de dichos estudiantes a los centros de Educación Secundaria se ha notado en las cifras del centro educativo. Por último y atendiendo a las matrículas de Educación Infantil cabe señalar que tan sólo aparece un alumno nuevo de nacionalidad marroquí.

Respecto a la plantilla escolar del centro para el curso 2008/2009 fue de 23 profesores, de los cuales 4 son hombres y 19 mujeres más una profesora no funcionaria de Religión. El centro cuenta además con una educadora y un miembro de personal de administración que hace funciones de monitora escolar. 
En último lugar, cabe resaltar los Planes y proyectos educativos en los que se encuentra inmerso el centro citado para el curso académico 2008/2009. Son los siguientes: Proyecto Comunidad de Aprendizaje; Programa de Calidad y Mejora; Proyecto para la incorporación de las Tecnologías de la Información y la Comunicación (TIC) a la Educación y la Gestión del Centro; Plan de Apertura, consistente en la oferta de servicios de Aula Matinal, Comedor y Actividades Extraescolares; El Deporte en la Escuela; Aprende a Sonreír (programa de higiene bucodental); Plan de Acompañamiento Escolar (clases de refuerzo educativo en horario extraescolar para los cursos de $3^{\circ}, 4^{\circ}, 5^{\circ}$ y $6^{\circ}$ ); Proyecto Intercultural. Aula ATAL. Programas de refuerzo del aprendizaje del español y de cultura materna y taller de cultura y lengua árabe; Diseños $\mathrm{Cu}$ rriculares de Educación Infantil y Primaria; Proyecto Lingüístico de Centro. Plan de Lectura y Biblioteca Enfoque comunicativo de la lengua. El Periódico de la Zona. PIN “La Radio Escolar”; Propuestas de Mejora tras los resultados de las Pruebas de Evaluación diagnóstica; y Escuela Espacio de Paz.

Destacar que desde el curso académico 2006/2007, este centro es Comunidad de Aprendizaje (CdA), lo cual ha supuesto un proyecto de transformación de sus estructuras y quehaceres pedagógicos que suponen potenciar y fortalecer las relaciones del centro educativo con las familias y la comunidad.

Siguiendo el trabajo desarrollado por el Grupo Inter (2006), los beneficios de trabajar con las CdA se encauzan en los siguientes ámbitos: a) para el alumnado: se logran mejores resultados académicos, actitudes y comportamientos, se disminuye el absentismo escolar; $b$ ) para las familias: los padres y madres o tutores del alumnado valoran más los procesos educativos, los profesores valoran más a las familias, los padres tienen mayor confianza a la hora de pensar en el trabajo que los profesores hacen con sus hijos, se da mayor continuidad y coherencia en el trabajo que hacen familia y escuela; $c$ ) para los profesores: la motivación e implicación del profesorado mejora, las familias valoran más a los profesores/as y la percepción de la escuela mejora en la comunidad.

Colegio de Jaén. Este colegio público está enmarcado en un barrio obrero de clase social media y media baja. El centro es uno de los primeros edificios públicos de esta parte de la ciudad que se construyó en la década de 1950 para atender las necesidades de escolarización del incipiente barrio de aquella época. Se encuentra enclavado frente al mercado del mismo nombre en un área mixta de carácter residencial y comercial.

En términos educativos, en el curso 2008/2009 cuenta con un total de 360 alumnos, de los cuales 39 tienen una nacionalidad distinta a la española. Aunque es conveniente destacar que existe un alto porcentaje de alumnado 
que si bien ha nacido en España es hijo/a de padres de otras nacionalidades. Si hacemos una distinción según país de nacimiento del alumnado obtenemos que 321 alumnos han nacido en España, 12 en Marruecos, 9 en Colombia, 8 en Ecuador, 4 en Rumania, 2 en Venezuela, 1 en Bolivia, 1 en la India, 1 en Italia y 1 en Pakistán. Tiene una proporción del $20 \%$ de alumnado de familias que provienen de algún país distinto a España.

Por otra parte y respecto al alumnado de Educación Especial de Integración, cabe señalar que existen 10 alumnos para el curso 2008/2009. La plantilla escolar del centro para el último curso mencionado es de 31 profesores y monitores. El Claustro de profesores actualmente tiene el siguiente perfil administrativo: 17 profesores definitivos en el centro, 2 profesores en comisión de servicio, 7 profesores con destino provisional, 1 profesor en fase de prácticas, 1 profesor de religión católica, 1 monitor de educación especial y 1 monitor de administración y servicios.

Por último, nos parece importante incluir los Planes y Programas que se desarrollan en el centro educativo que nos ocupa para el curso 2008/2009. Son los que siguen: Plan de Coeducación; Plan de Compensación Educativa; Proyecto de Apertura; Programa de Aplicación de las TIC; Programa de bilingüismo; Programa "El Deporte en la Escuela"; Programa de Acompañamiento; Plan de Autoprotección; Proyecto Escuela Espacio de Paz; Programaciones; y Atención a la diversidad.

A diferencia del colegio sevillano, el abordaje de la diversidad -relacionado con la inmigración- es una cuestión que está incorporada de forma tangencial en programas educativos diversos y de forma discontinua, pero no supone una transformación de la estructura organizativa.

Descrito en términos generales el marco empírico-contextual de la investigación desarrollada, este artículo responde al objetivo principal de analizar el modo en el que las familias -tanto autóctonas como extranjeras- participan en el ámbito escolar, reflexionando sobre las diferentes casuísticas que impiden su presencia más "normalizada" en el centro educativo, así como conocer las actividades organizadas desde los colegios que intentan promover la participación familiar. Todo ello atendiendo a la perspectiva del profesorado en torno a la presencia familiar en la escuela y con un eje transversal: "lo intercultural".

Técnicas de recogida de datos

En el tercer periodo de investigación -donde se inscriben los datos en los que se basa el contenido de este artículo- desarrollado durante el curso académico 2010-2011, se realizaron entrevistas etnográficas (Velasco y Díaz de Rada, 
1997) tanto formales como informales a profesorado de Educación Primaria, así como a familias tanto autóctonas como extranjeras, incluyendo aspectos a partir de los cuales iniciar una conversación donde la persona entrevistada pudiera desarrollar un discurso.

En las entrevistas dirigidas al profesorado se abordaron temas relevantes -la descripción del centro escolar donde trabajan, las situaciones difíciles de resolver que se encuentran en la práctica educativa, el modo como interpretan qué es la diversidad, las maneras de abordar la interculturalidad, las habilidades y destrezas competenciales que manejan y/o debieran manejar o sus demandas formativas, indagando sobre el peso atribuido a las familias en el papel educador, la relación que existe con el centro, sus formas de participación, su intervención en las Asociaciones de Madres y Padres de alumnos/as (en adelante, AMPAs), una mirada comparativa entre familias españolas y extranjeras, etc.-, en el marco de las siguientes dimensiones: a) Descripción general del centro; b) Programas de atención a la diversidad; c) Alumnado: proceso de escolarización, relaciones socioeducativas; y d) Relaciones del centro con las familias.

En cuanto a las entrevistas dirigidas a las familias, en éstas se abordaron temas -los criterios seguidos en el proceso de elección del colegio, el transcurso de recepción, sobre cómo se encuentra su hijo/a en el colegio y tipo de actividades escolares y extraescolares en las que está implicado/a, sobre el papel que consideran debe tener la familia en el proceso formativo del menor y el que debe tener el sistema educativo, su valoración sobre la presencia de alumnado de origen extranjero (en el caso de familias españolas), sobre cómo se siente su hijo/a (en el caso de ser de origen extranjero) y tipo de respuestas que han llevado a cabo para incorporarles, sobre su implicación en actividades escolares, etc. - que configuran las dimensiones: a) Características de la unidad familiar; $b$ ) Proceso de escolarización; $c$ ) Papel de la familia en la escuela; y d) Relaciones escuela-familia.

Respecto a los informantes participantes, cabe indicar que han sido un total de 24, divididos de la siguiente forma: cuatro profesores/as de cada uno de los centros, cuatro familiares extranjeros de cada uno de los centros y cuatro familias autóctonas en cada uno de los centros (véase la Tabla 1). ${ }^{1}$

En cuanto a los criterios seguidos para la selección de los informantes, éstos fueron:

1 Esta tabla se encuentra al final del presente documento, en el Anexo (Nota del Editor). 
- Para el caso del profesorado: miembros del equipo directivo, ya que nos aportarían una visión amplia del funcionamiento y la casuística del centro educativo en cuanto a nuestro objeto de interés; profesores de apoyo y profesores en general que mostraran disponibilidad.

- Para el caso de los familiares: miembros del Consejo Escolar o la Asociación de Padres y Madres de alumnos/as del centro y disponibilidad.

Procedimiento de análisis de datos

El objetivo fundamental del proceso de análisis de datos ha sido el de buscar significado y aportar reflexividad de los discursos emitidos por parte de los actores sin perder de vista precisamente los contextos en los que éstos se producían. De este modo, el interés último de esta investigación se enmarca en los principios epistemológicos y metodológicos de la Antropología, en términos de Geertz (2005): aportar interpretaciones de los significados que manejan las personas.

Para seguir un planteamiento analítico de la información de forma rigurosa y científica procedimos a utilizar un sistema de tratamiento de la información ordenado y sistemático. Para organizar y analizar la información se ha tenido como herramienta fundamental el programa informático de tratamiento de datos cualitativo QSR NVIVO8.

Los pasos fueron:

- Transcripción de las entrevistas. Toda la información almacenada a través de la grabadora fue trascrita respetando la literalidad del discurso emitido. Estos documentos pasaron a formar parte de los recursos internos con los que trabajaríamos a través del programa QSR NVIVO8, siendo organizados según contextos donde se recopilaron (Jaén o Sevilla) y según actores entrevistados (profesorado y familias).

- Definición de ejes analíticos. En segundo lugar, se redujo toda la información en unidades de significado para facilitar su manejo para la interpretación. Se estableció así una serie de ejes analíticos, "unidades de contenido" según Werner y Schopfle (1987), que han permitido ordenar la información recopilada. Son los que siguen: a) homogeneidad versus heterogeneidad; b) reconocimiento y/o problematización de la diversidad; $c$ ) lenguas; $d$ ) racismo; $e$ ) signos de cuestionamiento de lo que hacemos y decimos y $f$ ) estructura y organización escolar. 
- Codificación e interpretación de los datos. En tercer lugar, se procedió a codificar los datos obtenidos en función de los ejes analíticos y a su posterior interpretación.

\section{Análisis de resultados}

Al tratar de abordar temáticas relacionadas con el trinomio escuela, inmigración y familia, surgen -con base en los datos recopilados en este trabajo de investigación- diferentes ámbitos y niveles de análisis. Nos centramos, a continuación, en los términos en los cuales aparece la familia en esta amalgama educativa y que tiene que ver con "lo intercultural".

En este sentido, se analizará el nivel de participación de las familias tanto autóctonas como extranjeras en el centro escolar donde se encuentran escolarizados/as sus hijos/as, así como el tipo de actividades que se organizan desde los centros educativos y en los cuales las familias tienen una presencia importante.

\section{Participación de las familias en la escuela, familias extranjeras en particular}

En cualquier contexto democrático, hablar y reflexionar sobre la participación es una cuestión básica y fundamental. En los contextos educativos, ésta debe aparecer igualmente esencial. Además de una respuesta democrática, la participación familiar en los centros educativos supone una necesidad primordial para el adecuado desarrollo socioeducativo de los/as hijos/as escolarizados/as. Sin embargo, la reflexión sobre esta cuestión no escapa a dificultades.

Para el análisis de la participación y el estudio de las relaciones que existen entre la institución escolar y la familia habría que detenerse en algunos factores: uno de ellos surgiría, como punto de partida más teórico, en el propio significado de participación y qué se entiende por este concepto, quiénes lo definen entre los diferentes agentes y/o actores del escenario educativo. Otro de los factores a tener en cuenta sería la reflexión sobre el canal de comunicación y los recursos utilizados para acercar el colegio y la vida cotidiana de éste al mundo familiar. Otra cuestión a considerar sería el nivel de expectativas de las familias hacia el colegio y sobre todo hacia aquello que se organiza desde el mismo. Un factor más sobre el cual reflexionar supondría detenerse en aquellos aspectos a los que se dirige la participación y aquellos otros en los que no se participa ni se incita a ello, entre otros muchos factores más. 
(...) la escuela tiene una función educativa muy importante, pero la escuela si no va acompañada del refuerzo en casa, entonces en la escuela no podemos hacer nada. La educación de los niños tiene que ser una labor conjunta (profesor/a de educación primaria de Jaén, 2009).

Siguiendo las indicaciones de Pozuelos (2008) y teniendo en cuenta la investigación desarrollada podemos destacar que en el caso del CEIP de Sevilla existe una participación importante de las familias en el ámbito didáctico de la institución escolar, dada la particularidad de dicho centro al constituir una Comunidad de Aprendizaje. Dando un paso más allá, además existe una integración real de población inmigrante que es considerada como un factor positivo entre el resto de familias. En el siguiente testimonio de una madre española representante del Consejo Escolar se puede comprobar:

Entrevistadora: ¿Qué te ha parecido que este año entrara Fatiha en el Consejo Escolar?

Entrevistada: Me parece muy bien.

Entrevistadora: ¿Cómo crees que ha pasado eso?

Entrevistada: Porque está bien, ipor qué no va a entrar una musulmana leñe! Si la muchacha está implicada en todo, pobrecita. Cuando hay que hacer los pasteles, corriendo vienen, cuando vinieron los del Almería, corriendo vinieron a la reunión, que son gente que se brinda, que vienen a todas las reuniones y están dando la cara, que por ser musulmana también tienen derecho (familiar de Sevilla, 2009).

Es cierto que destacamos el CEIP sevillano, porque es el único que se ha encontrado donde las familias se hallan implicadas en aspectos más puramente didácticos de la educación de sus hijos/as en el contexto escolar. En el resto de experiencias, la participación está ligada netamente a actividades extraescolares -no por ello menos importantes-.

Una de las cuestiones que parecen claves para pensar sobre participación familiar en los centros educativos es tener en cuenta la opinión de los padres acerca del funcionamiento del colegio y los profesionales que intervienen en él. En este sentido, los datos demuestran que si existe una buena opinión por parte de las familias sobre el colegio y todo aquello que se organiza desde el mismo, la participación estará más alentada y se producirá más naturalmente que si, por el contrario, existen importantes discrepancias entre el centro educativo y la familia.

El siguiente testimonio de un padre español de Jaén es un claro ejemplo de una disconformidad absoluta con las actividades extraescolares en el centro y la organización de las mismas, lo que lleva consigo una escasa participación: 
Entrevistadora: ¿Tus hijos asisten a actividades extraescolares en el centro?

Informante: Van a actividades fuera del centro. Los tuve apuntados a actividades dentro del centro el año pasado, pero si te digo la verdad pues yo creo que allí iban a perder el tiempo, vamos no hicieron nada. Empezaron en informática y no hicieron nada, luego se apuntaron a deporte en la escuela y a esto tampoco se le vio color. Yo lo he intentado porque a mí me gusta que el centro funcione y que giren en este tipo de actividades, pero es que no están bien organizadas y van a perder el tiempo (familiar de Jaén, 2009).

Sin embargo, y en contraposición a dicho argumento, a lo largo de esta investigación hemos constatado, en mayor medida, numerosos discursos de familias que expresan estar de acuerdo con las directrices llevadas en el centro, teniendo muy buena opinión sobre el profesorado, tal y como manifiesta una madre española de Jaén:

(...) los profesores no se echan atrás, o sea que son muy dispuestos a emprender y a hacer cosas nuevas (...) surge lo del bilingüismo allá que se apuntan, surge otro proyecto de coeducación también, que tiene que ir a clases de Inglés allá que van (...) son personas muy participativas (...) veo este centro muy positivo por el profesorado tan implicado y activo (familiar de Jaén, 2009).

A pesar de la buena sintonía entre la familia y el centro educativo, la participación, en términos generales, es descrita por los diferentes agentes del medio educativo como escasa. Los discursos que se refieren a la participación de las familias -tanto extranjeras como nacionales- en las diferentes actividades extraescolares que el centro educativo organiza resaltan dos factores entre los cuales pivota, de alguna forma, la justificación de la escasa participación en las mismas.

Uno de ellos radica en la cuestión económica y las posibilidades que ello ofrece o no para el pago de la cuota en el caso del AMPAs o de las actividades puntuales que se desarrollan. A su vez, este factor aparece también relacionado con el tiempo disponible de las familias para hacerse presentes en el centro y acompañar a sus hijos en las actividades. En el siguiente testimonio procedente de una madre española, miembro del AMPA, se puede apreciar esta idea:

(...) aquellas actividades que son un poco caras, pues no se hacen porque nadie va a poder pagarlas. Entonces tenemos que tener en cuenta a la hora de hacer las actividades, pues las capacidades económicas de las familias. Por ejemplo, a las granjas escuela no se suele ir porque vale mucho dinero, pensamos que estos viajes son muy caros para estos niños, y se buscan otras actividades (familiar de Jaén, 2009).

$\mathrm{El}$ otro factor que encontramos -sin diferenciar entre familias nacionales y extranjeras- se refiere al interés que las familias puedan mostrar hacia la vida extraescolar de los centros educativos y la asistencia a las actividades que se planifican; el siguiente testimonio de una madre española, miembro del AMPA, es un ejemplo de ello: 
La información que da el colegio es buena, el AMPAs hace una reunión general al principio de curso. Y a esta reunión siempre hemos venido como máximo 15 o 20 personas, aunque este año ya fue increíble porque es que estábamos sólo los que estamos en el AMPAS, vamos cinco personas de todo el colegio (...) ha habido pocas asistencias (...) Y esto es todo la falta de interés de los padres (familiar de Jaén, 2009).

A su vez, dicho interés aparece también directamente relacionado con la forma de entender dichas actividades, y las finalidades y expectativas con las que las familias y sus hijos/as pueden acudir a ellas. Y es aquí donde encontramos dos tipos de argumentos, uno que comparte una mirada hacia las actividades extraescolares como entretenimiento para los/as niños/as y el centro como lugar donde éstos/as pueden estar mientras termina la jornada laboral de las familias, tal y como explica una madre de origen marroquí de Jaén:

Entrevistadora: ¿Ellos están apuntados a alguna actividad extraescolar por las tardes en el colegio?

Informante: No.

Entrevistadora: ¿Por qué?

Informante: Porque no los he apuntado, porque se quedan en casa todos los días. Porque lo apuntas, pagar más dinero.

Entrevistadora: Así estás tú con ellos.

Informante: Claro, si no tenía otro remedio, si tengo trabajo lo apuntamos, si no tengo trabajo y me quedo en casa para qué los apunto (familiar de Jaén, 2009).

El otro argumento al respecto deja ver una función formativa y de enriquecimiento en las actividades que desde el centro se organizan y, por tanto, la necesidad de participar en ellas, tal y como indica una madre española de la ciudad de Sevilla: “(...) todo lo que hagan aquí para que se diviertan los niños, para que se impliquen, para que conozcan, para que vengan los padres" (familiar de Sevilla, 2009).

Concluyendo sobre el factor interés de las familias por participar en lo que acontece en los centros educativos de sus hijos/as, éste no se muestra dependiente de la extranjeridad o no de las familias, sino más bien responde a una cuestión más personal que no atiende al origen de los núcleos familiares y así lo comunica una madre española de Jaén: “(...) yo creo que da igual que sea extranjero o español, esto va en que las personas tienen poco interés y esto no es porque sean inmigrantes" (familiar de Jaén, 2009).

Lo cierto es que los discursos recogidos muestran que las familias -sobre todo extranjeras - tienen conocimiento de la existencia del AMPA en los centros educativos, lo cual indica que la posible falta de participación de las familias 
no responde a un desconocimiento de los aspectos organizativos o funcionales de los colegios en lo referente a esta cuestión; tal y como se puede comprobar en el siguiente argumento de una madre de origen marroquí de Jaén:

Entrevistadora: ¿Sabes que existe en el colegio una asociación de padres y madres AMPAs?

Informante: Sí.

Entrevistadora: ¿Y participas en el AMPAs?

Informante: Sí pero con dinero.

Entrevistadora: ¿`Y asistes a las reuniones del AMPAs?

Informante: He venío a dos y a la otra no puedo vení. Luego tengo mucho trabajo en la casa y he venío a do y ya está (familiar de Jaén, 2009).

Incluso el siguiente discurso de un profesor de un centro educativo jiennense resalta la buena relación existente entre el AMPA y el colegio: "Pues esas actividades son importantísimas, eh, en este colegio yo es que estoy que alucino con la buena relación que hay con el AMPAs, con la buena marcha del Consejo de Dirección (...) los padres de la asociación responden perfectamente, los padres del Consejo Escolar responden de maravilla”. Aunque igualmente indica la escasa participación de los padres en términos generales en las actividades del centro:

(...) Pero debo decir que en general la participación es pobre. Yo desearía que los padres en general, pues respondieran mejor a estas actividades. Yo no sé si habría que hacer también más publicidad, igual podríamos hacer como una especie de difusión, a lo mejor es cuestión también de la publicidad que se le da. Luego después de la actividad pues también hacer algunas actividades, cómo lo pasasteis, que hicimos, preparar fotos. Puede que sí que en esto podríamos mejorar bastante en la participación se podría mejorar (profesor/a de educación primaria de Jaén, 2009).

Como queda patente en este último fragmento de la respuesta de un profesor, existe disposición y preocupación por parte de este sector por la mejora y el aumento de la participación familiar en el centro educativo, y, en este sentido, se reflexiona y se buscan posibles actuaciones que puedan mejorarla: dar mayor publicidad e información sobre lo que se organiza y realizar una evaluación de las actividades para conocer el grado de satisfacción de las familias con aquello desarrollado; así como concretar las actividades mucho más a nivel de aula y no involucrando a todo el colegio:

Había una profesora que en vez de hacerlo a nivel general lo que hacía es organizar una merienda, y venían todos los padres. Y como había gente de otros países, pues traían dulces típicos de otros países. Y si que es verdad que participaban más que a nivel de colegio. A nivel de colegio es algo más disperso y se pierde (familiar de Jaén, 2009). 


\section{Actividades extraescolares interculturales}

A lo largo de este periodo de investigación y nuestro contacto con los centros educativos hemos constatado que las actividades extraescolares que se organizan y se ponen en práctica en los centros y en donde la presencia de las familias es más o menos visible giran, en términos generales, en torno a diferentes temáticas:

a) En unos casos tienen como objetivo la celebración de algún día específico que coincide, en la mayoría de los casos, con alguna fecha festiva: Día de la paz, Día de Andalucía, Día del nuevo año chino, Navidad, carnavales, fin de curso, etcétera.

b) En otros casos, se organizan y desarrollan celebraciones interculturales que se dedican al encuentro cultural entre las diferentes familias que tienen escolarizados/as a sus hijos/as en el centro, con una especial mirada hacia las familias extranjeras.

(...) Lo pasamos muy bien, una merienda muy grande, ha venido tres mujeres en mi casa y la hemos hecho en mi casa. Que aquí se celebra todo de Rumanía, de China y bien, con la directora con todo bien. Muchas madres, españolas, todas. Fue una merienda muy grande, la mesa grande llena de eso. El día de la fiesta como había muchas personas no ha sobrado ni un poquito (familiar de Sevilla, 2009).

Como puede comprobarse en este último discurso de una madre de origen rumano, las actividades extraescolares en las que se solicita una mayor participación por parte de las familias tanto en la preparación como en el desarrollo de las mismas, son bien recibidas por parte de los padres y madres. Al mismo tiempo, existe una demanda explícita por parte de las familias para la organización de actividades festivas que muestren y recreen parte de la "cultura" de origen de las familias extranjeras que se encuentran en el centro. En el siguiente discurso de un padre colombiano que tiene su hijo escolarizado en un centro educativo de Jaén se hace patente:

Informante: (...) Hombre, yo opino que el año es muy largo, el año tiene mucho días y por ejemplo, una vez al año decir cuántos ecuatorianos, y hacer una actividad de su país. Entonces yo por ejemplo, los colombianos decimos pues mira, una actividad colombiana, que ilusión y la hacemos. Los ecuatorianos otro día, los indios otro día, pero claro, si no hay mucho indio... es que ese es el problema, que si no hay muchos ¿qué actividad pueden hacer?

Entrevistadora: ¿Entonces crees que deberían de hablar más de las culturas y hacer días específicos?

Informante: Claro, hacer actividades. También depende de la cantidad de personas que haya. Si hay una persona, ¿qué clase de actividades puede hacer una sola persona? Si hay 
muchos más colombianos, ecuatorianos, peruanos, pues también ahí está el problema (...) pero por lo menos dar oportunidades que muestren la cultura y que muestren las actividades que se pueden hacer de ese país. Por ejemplo, que hagan una actividad española de las actividades españolas que se puedan hacer y dejar a los colombianos detrás, hacer una colombiana en el mismo día, pero mostrar un poquito de cada país y de cada cultura (familiar de Jaén, 2009).

Como vemos, el objetivo último en la demanda de este tipo de actividades "más interculturales" es acercar, aproximar e integrar las "culturas" presentes en el centro educativo, dejando un espacio para mostrar aspectos sustantivos de tales "culturas". Ahora bien, pese a la aceptación del centro y las familias de este tipo de prácticas, desde nuestro posicionamiento teórico rebatimos de alguna manera su realización, ya que responden a la "cosificación", "esencialización" y "folklorización" de "la cultura" del país de procedencia de las familias y alumnado. Puede suponer un aumento de las distancias culturales ya existentes que deben venir acompañadas de un tratamiento más didáctico de estas temáticas, con el fin de no fomentar las diferencias que ello puede provocar de forma, por supuesto, no intencionada por parte de los diferentes agentes involucrados.

\section{Conclusiones}

Con este trabajo se evidencia una vez más que la escuela y la familia deben trabajar de forma paralela, ya que ambos son agentes encargados y responsables de la formación de sus hijos/as, de transmitir valores y educar; y hacerlo de forma coordinada, se entiende, repercute de forma positiva en el propio alumnado.

En este trabajo se ha puesto el acento sobre uno de los agentes activos en el espacio escolar: la familia; analizando la participación de la misma en las dinámicas escolares formales y focalizando la mirada reflexiva hacia las familias extranjeras, ya que, a priori, son caracterizadas como culturalmente diferentes y con problemáticas añadidas que tendrían algo que decir sobre los niveles de comunicación y participación en la escuela.

Con base en el estudio realizado cabe resaltar que la toma de contacto y tipo de relación establecida entre el profesorado y las familias de los/as menores escolarizados/as se inscribe en el territorio de la escuela a partir de lógicas y prácticas de participación e implicación escolar que tienen que ver con los grados de una determinada tradición institucional que lo posibilita o lo dificulta, la sensibilidad de las/as profesionales educativos para crear espacios de comunicación y encuentro, los tipos de familia y su interés/disponibilidad hacia la práctica educativa, entre otros factores. 
A lo largo de estas páginas y a la luz de los resultados empíricos obtenidos se puede concluir que la participación de las familias en los centros educativos no depende directamente de la extranjeridad de las mismas. A su vez, es el interés por la educación y la escuela el núcleo principal que hace depender, en mayor medida, el nivel de participación de las familias en los centros educativos.

\section{Bibliografía}

Aguado, Teresa (1996), Educación Intercultural. Su teoría y su práctica, Madrid: UNED.

Beck, Ulrico (1998), La sociedad del riesgo: hacia una nueva modernidad, Barcelona: Paidós. Bertely, M. (2001), Conociendo nuestras escuelas. Un acercamiento etnográfico a la cultura escolar, México D.F.: Paidós.

Colectivo IOE (2007), Inmigración, Género y Escuela, Madrid: Ministerio de Educación y Ciencia. De la Guardia, R. M. y F. Luengo (2007), “Las relaciones con las familias y con el entorno”, en A. Bolívar y A. Guarro [coords.], Educación y cultura democráticas, Madrid: Wolters Kluwer.

Essomba, M. A. (2008), 10 ideas clave. La gestión de la diversidad cultural en la escuela, Barcelona: Graó.

García Borrego, I. (2008), Herederos de la condición inmigrante: adolescentes y jóvenes en familias madrileñas de origen inmigrante, Tesis doctoral inédita, Madrid: Departamento de Sociología, UNED.

García Castaño, F. J., Rubio Gómez, M.y O. Bouachra (2008), "Población inmigrante y escuela en España: un balance de investigación”, en Revista de Educación, núm. 345, España.

Geertz, C. (2005), La interpretación de las culturas, Barcelona: Gedisa.

González Barea, E. (2008), "Convivencia intercultural en centros educativos multiculturales... ¿Hacia un reconocimiento de la diversidad cultural?”, en Educatio Siglo XXI, vol. 26, España.

Gorski, Paul C. (2009), "Intercultural education as social justice", en Intercultural Education, vol. 20, núm. 2, London.

Grupo Inter (2006), Culture is ourfocus, diversity i sour normality. INTER guide to implement intercultural education, Viena: Navreme.

Hernández Prados, M. A. y H. López Lorca (2006), "Análisis del enfoque cultural de la cooperación padres y escuela", en Aula abierta, núm. 87, España.

Nieto, Sonia (1992), Affirming diversity. The sociopolitical context for Multicultural Education, Nueva York: Longman.

Ogbu, J. U. (1993), "Etnografía escolar. Una aproximación a nivel múltiple", en Velasco, H. M., García Castaño, F. J. y A. Díaz de Rada [eds.], Lecturas de antropología para educadores, Madrid: Trotta.

Pozuelos, F. J. (2008), "Metodología didáctica: el currículum en el aula de educación primaria”, en A. De la Herrán y J. Paredes [coords.], Didáctica General, Madrid: McGraw-Hill.

Rodríguez Izquierdo, R. M. (2011), "Discontinuidad cultural. Estudiantes inmigrante y éxito académico", en Aula Abierta, vol. 1, núm. 39, España.

Velasco, H. y Díaz de Rada, A. (1997), La lógica de la investigación etnográfica, Madrid: Trotta.

Werner, O. y G. M. Schoeple (1987), Systematic fieldwork. Volume 1. Foundations of ethnography and interviewing, London: Sage Publications.

Wolcott, Harry F. (1993), “Sobre la intención etnográfica”, en Velasco, H., García, F.J. y A. Díaz de Rada [eds.], Lecturas de antropología para educadores. El ámbito de la educación $y$ de la etnografía escolar, Madrid: Trotta. 
Anexo

Tabla 1

\begin{tabular}{lll}
\hline $\begin{array}{l}\text { Colegio/ } \\
\text { Informante }\end{array}$ & Sevilla & Jaén \\
\hline Profesorado & -Profesora directora & \\
& -Profesora jefa de estudios & -Profesor director \\
& -Profesora ATAL & -Profesor jefe de estudios \\
& -Profesora de infantil & -Profesor apoyo primaria \\
\hline Familiares & -Madre & -Profesor de primaria \\
autóctonos & -Madre & -Madre \\
& -Madre & -Madre \\
& -Madre & -Madre \\
\hline Familiares inmi- & -Madre marroquí & -Padre \\
grantes & -Madre boliviana & -Madre marroquí \\
& -Madre rumana & -Madre marroquí \\
& -Madre rumana & -Madre ecuatoriana \\
\hline
\end{tabular}

Fuente: Elaboración propia.

Eva María González Barea. Licenciada en Pedagogía y Doctora en Antropología Social y Cultural por la Universidad de Granada. Es profesora e investigadora en la Universidad de Murcia. Miembro del Grupo de Investigación Equidade Inclusión en Educación. Ha participado en diversos proyectos de investigación -tanto nacionales como internacionales- relacionados con educación, minorías étnicas, interculturalidad, género y diversidad, exclusión, etc. En tales temáticas continúa tanto con sus investigaciones como con sus publicaciones, de las que se destacan: Puerta, S. y González Barea, E., "Reproducción de los estereotipos de género en Educación Infantil a través de los juegos y juguetes", en Revista Investigación en la Escuela, vol. 85 (2015); González Barea, E. M., López Calvo, A. y Bernal Galindo, R., El currículo y la enseñanza, ¿qué se les enseña y cómo? (http://www.ugr.es/local/recfpro/rev133ART6.pdf). Profesorado. Revista de Curriculum y Formación del Profesorado, vol. 17 (2010).

Recepción: 20 de enero de 2015.

Aprobación: 15 de febrero de 2016. 
